\title{
The role of ERK5 in endothelial cell function
}

Gopika N. Nithianandarajah-Jones*, Bettina Wilm†, Christopher E.P. Goldring*, Jürgen Müllerł and Michael J. Cross ${ }^{\star 1}$

*MRC Centre for Drug Safety Science, Department of Molecular and Clinical Pharmacology, Institute of Translational Medicine, University of Liverpool, Liverpool L69 3GE, U.K. †Department of Cellular and Molecular Physiology, Institute of Translational Medicine, University of Liverpool, Liverpool L69 3GE, U.K.

¥Warwick Medical School, University of Warwick, Coventry CV4 7AL, U.K.

${ }^{1}$ To whom correspondence should be addressed (email m.j.cross@liv.ac.uk).

Key words: angiogenesis, big MAPK1 (BMK1), endothelial cell, extracellular-signal-regulated kinase 5 (ERK5), mitogen-activated protein kinase (MAPK), signal transduction.

Abbreviations: BLMEC, bovine lung microvascular endothelial cell; DUSP, dual-specificity phosphatase; E, embryonic day; eNOS, endothelial nitric oxide synthase; ERK, extracellularsignal-regulated kinase; FGF2, fibroblast growth factor 2; HDMEC, human dermal microvascular endothelial cell; HUVEC, human umbilical vein endothelial cell; KLF, Krüppellike factor; MAPK, mitogen-activated protein kinase; MAPKK, MAPK kinase; MAPKKK, MAPKK kinase; MEF, myocyte enhancer factor; MEK, MAPK/ERK kinase; MEKK, MEK kinase; MKP, MAPK phosphatase; NF- $k \mathrm{~B}$, nuclear factor $k \mathrm{~B}$; NLS, nuclear localization signal; NRF2, nuclear erythroid 2-related factor 2; PKB, protein kinase B; RSK, ribosomal S6 kinase; SGK, serum- and glucocorticoid-regulated protein kinase; TNF $\alpha$, tumour necrosis factor $\alpha$; VCAM-1, vascular cell adhesion molecule 1; VEGF, vascular endothelial growth factor. 


\begin{abstract}
Extracellular-signal-regulated kinase 5 (ERK5), also termed big MAPK1 (BMK1), is the most recently discovered member of the mitogen-activated protein kinase (MAPK) family. It is expressed in a variety of tissues and is activated by a range of growth factors, cytokines and cellular stresses. Targeted deletion of Erk5 in mice has revealed that the ERK5 signalling cascade is critical for normal cardiovascular development and vascular integrity. In vitro studies have revealed that, in endothelial cells, ERK5 is required for preventing apoptosis, mediating shear-stress signalling and regulating tumour angiogenesis. The present review focuses on our current understanding of the role of ERK5 in regulating endothelial cell function.
\end{abstract}

\title{
Introduction
}

Mitogen-activated protein kinases (MAPKs) play an essential role in regulating many cellular processes including growth, differentiation and apoptosis. MAPKs are activated by a range of growth factors and chemical stimuli such as oxidative stress and osmotic imbalance, and are responsible for transducing extracellular signals to the cytoplasm and nucleus. In mammalian cells, the MAPK signalling system consists of four distinct linear signalling cascades terminating in: extracellular-signal-regulated kinase (ERK) 1 and 2, c-Jun N-terminal kinase (JNK) 1, 2 and 3, p38 MAPKs (p38 $\alpha, \beta, \gamma$ and $\delta$ ), or the most recently discovered MAPK, ERK5, also termed big MAPK1 (BMK1) [1,2]. Each of these terminal kinases phosphorylates a range of cellular targets from cytoplasmic enzymes to transcription factors [3].

\section{Structure of ERK5}

The human ERK5 gene (also termed MAPK7) is present on chromosome 17p11.2 and spans 5824 bases. It has an ORF of 2451 bp encoding a protein of 816 amino acids with a predicted molecular mass of $102 \mathrm{kDa}$ (Figure 1). ERK5 shares $66 \%$ sequence homology with ERK2 within the kinase domain, which contains the Thr-Glu-Tyr (TEY) dual phosphorylation motif in the activation loop [4]. The N-terminal domain (amino acids 1-406) of ERK5 contains a region important for cytoplasmic targeting (amino acids 1-77) and the kinase domain (amino acids 78-406), which can be further separated into regions required for interaction with MAPK/ERK kinase 5 (MEK5) (amino acids 78-139) and oligomerization (amino acids 140-406) [5]. The large size of ERK5 is attributable to its long C-terminal tail of 410 amino acids, which is unique among the MAPKs. The C-terminal domain contains a nuclear localization signal (NLS) (amino acids 505-539) situated between two proline-rich domains (amino acids 434-465, amino acids 578-701) that are proposed to serve as binding sites for Src homology 3 (SH3)-domain containing proteins $[4,5]$.The C-terminal region also contains a myocyte enhancer factor 2 (MEF2)-interacting region (amino acids 440-501) and a transcriptional activation domain (amino acids 664-789), which regulates MEF2 transcription factor activity [6]. Truncation of the C-terminal tail results in increased ERK5 kinase activity, revealing that the C-terminal tail of ERK5 also has an autoinhibitory function [7].

\section{Activation and regulation of the ERK5 signalling axis}

Activation of a MAPK signalling module is initiated by the activation of the apical MAPK kinase kinase (MAPKKK) by an extracellular stimulus, resulting in the sequential activation of the MAPK kinase (MAPKK) and ultimately the MAPK [8]. MEK5 is the upstream MAPKK that specifically phosphorylates ERK5 on Thr218/Tyr220 within the TEY motif in the activation loop, resulting in an increase in the catalytic activity of ERK5 $[4,9,10]$ (Figure 1). MEK5 is activated by the upstream MAPKKKs, MEK kinase 2 (MEKK2) and MEKK3, which specifically phosphorylate Ser313/Thr317 in MEK5 [11,12].

Active ERK5 is able to phosphorylate MEK5 [13] as well as autophosphorylate on a number of C-terminal tail residues, leading to an enhancement of ERK5 transcriptional activity [14]. More recent data have shown that ERK5 is able to undergo MEK5-independent phosphorylation by cyclin-dependent kinases (CDKs), revealing a potential novel mode of ERK5 activation $[15,16]$. Dephosphorylation of MAPKs on the Thr-Xaa-Tyr (TXY) motif by a MAPK phosphatase (MKP) subfamily of dual specificity phosphatases (DUSPs) leads to their inactivation [17]. Currently, 
no DUSP has been identified that dephosphorylates ERK5. However, ERK5 is dephosphorylated by the phosphotyrosine-specific phosphatase PTP-SL, which interacts with ERK5 and impedes its translocation to the nucleus [18]. ERK5 is also regulated by posttranslational modifications; it has recently been reported that ERK5 undergoes SUMOylation by small ubiquitin-related modifier 3 (SUMO3) on Lys6 and Lys22 following treatment with $\mathrm{H} 2 \mathrm{O} 2$ and advanced glycation end-products (AGE) in human umbilical vein endothelial cells (HUVECs) [19].

Similar to the other MAPKs, ERK5 belongs to a family of evolutionarily conserved prolinedirected protein kinases that phosphorylate substrates on serine and threonine residues immediately preceding a proline residue. However, certain serine and threonine autophosphorylation sites in ERK5 are not followed by proline $[13,14]$, suggesting that the specificity of ERK5 may differ from other MAPK family members or that another kinase may be involved in the phosphorylation of these sites. Activation of the ERK5 signalling axis stimulates both distinct and similar pathways to the classical ERK1/2 pathway [20]. Downstream targets of ERK5 include the MEF family of transcription factors: MEF2A, MEF2C and MEF2D, Sap-1a (serum-response factor accessory protein 1a; ETS domain transcription factor), c-Myc, serum- and glucocorticoid-regulated protein kinase (SGK) and p90 ribosomal S6 kinase (RSK) (reviewed in [21,22]).

\section{The role of ERK5 in vivo}

To address the physiological role of the ERK5 signalling axis, researchers have utilized gene targeting in mice to ablate specific genes (for a detailed review, see [22,23]). Erk5 deficient mice die around embryonic day (E) 10.5 due to cardiovascular defects and angiogenic failure in embryonic and extra-embryonic tissues. In these mice, the developing vasculature fails to mature, with endothelial cells becoming disorganized and rounded leading to a loss of vascular integrity [24-27]. Similar phenotypic abnormalities are seen in mice lacking Mek5 [28] and Mekk3 [29], suggesting that the ERK5 signalling axis is critical to vasculogenesis and angiogenesis. In an attempt to determine the primary defect upon Erk5 gene ablation, researchers have generated conditional tissue-specific Erk5-knockout mice. Endothelial specific Erk5-knockout mice show cardiovascular defects and die around E10.0, similar to the conventional Erk5-knockout mice [27]. However, specific knockout of Erk5 in cardiomyocytes or hepatocytes does not affect development [27]. Recent studies using an inducible conditional knockout of Erk5 in neurogenic regions of the mouse brain have shown a role for ERK5 in neurogenesis [30]. Overall, the mouse data suggest that, whereas global Erk5 knockout affects cardiovascular development, the initial defect occurs in the endothelium and that ERK5 is critical for endothelial cell function. The requirement of ERK5 in the maintenance of vascular integrity is highlighted by the fact that induced ablation of Erk5 in adult mice is lethal within 23 weeks as blood vessels become leaky due to endothelial cell apoptosis [27].

\section{ERK5 and endothelial cell physiology}

Inhibition of endothelial apoptosis

As outlined above, targeted deletion of the ERK5 signalling axis in mice suggests that ERK5 plays an essential role in endothelial cell physiology by maintaining endothelial cell survival [22]. The requirement for ERK5 to protect endothelial cells from apoptosis was attributed to the necessity of MEF2C phosphorylation by ERK5 [27]; the phenotype of Mef2c - /- mice is similar to that of Erk5-/- mice, with embryonic lethality resulting from cardiac and vascular malformations [31-33]. However, it was observed that infection of Erk5-/- embryos with an adenovirus encoding a constitutively active Mef2c was only able to partially protect endothelial cells from apoptosis [27], suggesting the existence of effectors downstream of Erk5 that regulate apoptosis [34]. Recent in vitro studies using human dermal microvascular endothelial cells (HDMECs) demonstrated that ERK5 is required for vascular endothelial growth factor (VEGF)-mediated phosphorylation of protein kinase B (PKB)/Akt, resulting in increased expression of the pro-survival protein BCL-2 and increased phosphorylation of the pro- 
apoptotic protein BAD. This suppresses apoptosis, resulting in increased cell survival facilitating tubular morphogenesis of the endothelial cells in a collagen gel [35] (Figure 2).

A role for ERK5 in regulating PKB/Akt phosphorylation at Ser473 and Thr308 has previously been reported in mouse embryonic fibroblasts under conditions of osmotic stress [36]. In mouse neuronal cells, nerve growth factor (NGF) mediated phosphorylation of PKB/Akt on Ser473, but not Thr308, was dependent on ERK5 activity [37]. Furthermore, ERK5 has also been implicated in platelet-derived growth factor (PDGF)-induced activation of PKB/Akt in porcine aortic endothelial cells (PAEs) [38] and FLT-3-mediated activation of PKB/Akt in the $\mathrm{Ba} / \mathrm{F} 3$ pro-B-cell line [39]. Taken together, these data suggest that ERK5 may play a critical role in coupling growth factor receptors to activation of PKB/Akt and regulating cell survival; disruption of VEGF mediated PKB/Akt activation in endothelial cells may in part explain the profound vascular phenotype in the Erk5-/- mice. This raises the question of how ERK5 mediates PKB/Akt activation. It is unlikely that ERK5 directly phosphorylates PKB/Akt, as it is well established that PKB/Akt is activated via phosphorylation of Thr308 in the activation loop via the mammalian target of rapamycin complex 2 (mTORC2) [40,41], as well as Ser473 in the C-terminus by phosphoinositide-dependent kinase-1 (PDK1) [42]. It may be that ERK5 regulates the activity of a phosphatase, such as protein phosphatase 2 (PP2A) [43] or MKP3 [44], which have been shown to regulate PKB/Akt dephosphorylation. The precise mechanism through which ERK5 regulates PKB/Akt phosphorylation and activation remains obscure.

\section{Shear stress and atheroprotection}

Atherosclerosis is a condition in which patchy deposits of fatty material (atheromas or atherosclerotic plaques) develop in the walls of medium-sized and large arteries, leading to reduced or blocked blood flow. Investigations both in vivo and in vitro have shown that disturbed flow is proatherogenic by promoting oxidative and inflammatory states in the artery wall [45]. In contrast, steady laminar blood flow is atheroprotective by inhibiting oxidative stress and inflammation in the vessel wall $[45,46]$.

Fluid shear-stress-mediated ERK5 activation has been shown to confer an anti-apoptotic effect in bovine lung microvascular endothelial cells (BLMECs) [47] and an atheroprotective effect by negatively regulating tumour necrosis factor $\alpha$ (TNF $\alpha$ )-stimulated expression of adhesion molecules vascular cell adhesion molecule 1 (VCAM-1) and E-selectin in HUVECs [48]. A more recent study utilizing a novel MEK5 inhibitor, BIX02188, has revealed that the MEK5/ERK5 pathway mediates flow-dependent inhibition of TNF $\alpha$ signalling in BLMECs [49]. Analysis of laminar shear-stress-induced transcriptional responses in endothelial cells has identified Krüppel-like factor 2 (KLF2) as a mechanostress-induced gene [50,51], which is responsible for negatively regulating inflammation and angiogenesis and maintaining vascular quiescence [51-53]. KLF2 has subsequently been identified as an ERK5-responsive gene in mouse embryonic fibroblasts in a pathway requiring MEF2 transcription factor [54]. In addition, studies have shown that ERK5 is required for flow-induced expression of KLF2 in HUVECs [55] and human glomerular endothelial cells [56]. KLF2 inhibits activation of nuclear factor $k B$ (NF- $K \mathrm{~B}$ ) and subsequently reduces the expression of VCAM-1 and E-selectin on the vascular endothelium, which results in decreased adhesion of leucocytes and an anti-inflammatory response [51]. KLF2 is also implicated in up-regulating endothelial nitric oxide synthase (eNOS) and thrombomodulin expression in endothelial cells, which confer potent antithrombotic and anti-inflammatory properties resulting in a vasoprotective effect $[51,57]$. In addition to KLF2, ERK5 activation induces the expression of KLF4-dependent genes, which are also important in flow-mediated endothelial cell-protective responses [58,59] (Figure 2). Furthermore, statins have been shown to activate KLF4-dependent gene expression viaERK5in endothelial cells, suggesting that some of the pleiotropic vasoprotective effects of these drugs may be elicited through activation of ERK5 [58].

It has recently been observed that the flow-induced activation of ERK5 conveys a cytoprotective effect via activation of nuclear erythroid 2-related factor 2 (NRF2) in HUVECs [60]. NRF2 is a basic region leucine-zipper transcription factor which binds to the antioxidantresponse element (ARE) and thereby regulates the expression of a number of genes involved 
in the cellular antioxidant and anti-inflammatory defence as well as mitochondrial protection [61] (Figure 2).

\section{Tumour angiogenesis}

Angiogenesis is defined as the formation of new blood vessels from pre-existing vessels and plays a critical role both in normal physiological development and in the pathology of diseases such as cancer [62,63]. Hayashi et al. [27] initially reported that both VEGF and fibroblast growth factor 2 (FGF2) stimulate ERK5 activity in HUVECs and mouse lung capillary endothelial cells (MLCECs) [27], raising the possibility that ERK5 regulates angiogenesis in endothelial cells. More recent data have defined a role for ERK5 in regulating VEGF-mediated tubular morphogenesis in HDMECs, but not VEGF-mediated proliferation, in contrast with the role of ERK1/2, which regulates proliferation, but not tubular morphogenesis, in these cells [35]. Hayashi et al. [64] also provided initial evidence that ERK5 regulates tumour angiogenesis. Following the establishment of human melanoma and Lewis lung carcinoma tumour xenografts in mice, induced ablation of Erk5 in Erk5flox/flox mice carrying an inducible Mx1-Cre transgene resulted in a regression of the tumour vasculature and a concomitant reduction in tumour volume by $63 \%$ and $72 \%$ respectively [64]. The recent development of small-molecule inhibitors of MEK5 (BIX02188/BIX02189) [65] and ERK5 (XMD8-92) [66] is now allowing researchers to analyse the therapeutic effect of inhibiting the ERK5 signalling axis using in vivo animal models of tumour development. Pharmacological use of XMD8-92 in a mouse human tumour xenograft study revealed that tumour growth was inhibited by $95 \%$ and that FGF2-mediated angiogenesis was inhibited in Matrigel plugs [66]. This study also revealed that ERK5 is able to suppress the promyelocytic leukaemia protein (PML) in the nuclear body by direct phosphorylation of Ser403 and Thr409, consequently preventing the up-regulation of p21 expression, an important proliferation modulator [66]. Importantly, this study revealed that no adverse vascular effects were seen with XMD8-92 administration in mice, in contrast with those previously observed in the endothelium-specific deletion of Erk5 in mice leading to embryonic lethality [27]. This suggests that targeting ERK5 could be a viable anti-cancer therapeutic strategy. Indeed, the data discussed above would suggest that inhibiting ERK5 would simultaneously target both proliferation in cancer cells and VEGFstimulated tubular morphogenesis in endothelial cells, making this an attractive approach to targeting tumour growth and angiogenesis.

\section{Conclusions and perspectives}

It is now becoming apparent that ERK5, the most recently discovered MAPK, plays a critical role in cellular function. Whereas ERK5 appears to be almost ubiquitously expressed in different tissues, the phenotype of the ERK5-knockout mice indicates that it is critical for endothelial cell physiology. However, conditional knockout in other cell types suggests a degree of redundancy with other signalling pathways [22]. In vivo and in vitro studies have revealed that ERK5 is important for endothelial [23] and neuronal [37] cell survival, suggesting that, under certain conditions, these cell types have a critical dependence on ERK5 activity and may express specific ERK5 substrates not expressed in other cells. The recent development of small-molecule inhibitors of MEK5 and ERK5, combined with in vitro and in vivo animal models, will allow researchers to determine the roles of the ERK5 signalling axis in normal physiology and pathological conditions such as atherosclerosis and cancer.

\section{Funding}

We acknowledge funding from a Doctoral Training Grant (DTG) studentship from the Biotechnology and Biological Sciences Research Council (to G.N.N.-J. and M.J.C.), the Medical Research Council (to M.J.C. and C.E.P.G.) and the Wellcome Trust (to B.W.). 


\section{References}

1 Abe, J., Kusuhara, M., Ulevitch, R.J., Berk, B.C. and Lee, J.D. (1996) Big mitogen-activated protein kinase 1 (BMK1) is a redox-sensitive kinase. J. Biol. Chem. 271, 16586-16590

2 Chang, L. and Karin, M. (2001) Mammalian MAP kinase signalling cascades. Nature 410, $37-40$

3 Yang, S.H., Sharrocks, A.D. and Whitmarsh, A.J. (2003) Transcriptional regulation by the MAP kinase signaling cascades. Gene 320, 3-21

4 Zhou, G., Bao, Z.Q. and Dixon, J.E. (1995) Components of a new human protein kinase signal transduction pathway. J. Biol. Chem. 270, 12665-12669

5 Yan, C., Luo, H., Lee, J.D., Abe, J. and Berk, B.C. (2001) Molecular cloning of mouse ERK5/BMK1 splice variants and characterization of ERK5 functional domains. J. Biol. Chem. 276, 10870-10878

6 Kasler, H.G., Victoria, J., Duramad, O. and Winoto, A. (2000) ERK5 is a novel type of mitogen-activated protein kinase containing a transcriptional activation domain. Mol. Cell. Biol. 20, 8382-8389

7 Buschbeck, M. and Ullrich, A. (2005) The unique C-terminal tail of the mitogen-activated protein kinase ERK5 regulates its activation and nuclear shuttling. J. Biol. Chem. 280, 2659-2667

8 Widmann, C., Gibson, S., Jarpe, M.B. and Johnson, G.L. (1999) Mitogen-activated protein kinase: conservation of a three-kinase module from yeast to human. Physiol Rev. 79, 143180 PubMed

9 Lee, J.D., Ulevitch, R.J. and Han, J. (1995) Primary structure of BMK1: a new mammalian map kinase. Biochem. Biophys. Res. Commun. 213, 715-724

10 English, J.M., Vanderbilt, C.A., Xu, S., Marcus, S. and Cobb, M.H. (1995) Isolation of MEK5 and differential expression of alternatively spliced forms. J. Biol. Chem. 270, 28897-28902

11 Chao, T.H., Hayashi, M., Tapping, R.I., Kato, Y. and Lee, J.D. (1999) MEKK3 directly regulates MEK5 activity as part of the big mitogen-activated protein kinase 1 (BMK1) signaling pathway. J. Biol. Chem. 274, 36035-36038

12 Sun, W., Kesavan, K., Schaefer, B.C., Garrington, T.P., Ware, M., Johnson, N.L., Gelfand, E.W. and Johnson, G.L. (2001) MEKK2 associates with the adapter protein Lad/RIBP and regulates the MEK5-BMK1/ERK5 pathway. J. Biol. Chem. 276, 5093-5100

13 Mody, N., Campbell, D.G., Morrice, N., Peggie, M. and Cohen, P. (2003) An analysis of the phosphorylation and activation of extracellular-signal-regulated protein kinase 5 (ERK5) by mitogen-activated protein kinase kinase 5 (MKK5) in vitro. Biochem. J. 372, 567-575

14 Morimoto, H., Kondoh, K., Nishimoto, S., Terasawa, K. and Nishida, E. (2007) Activation of a C-terminal transcriptional activation domain of ERK5 by autophosphorylation. J. Biol. Chem. 282, 35449-35456

15 Diaz-Rodriguez, E. and Pandiella, A. (2010) Multisite phosphorylation of Erk5 in mitosis. J. Cell Sci. 123, 3146-3156

16 Inesta-Vaquera, F.A., Campbell, D.G., Tournier, C., Gomez, N., Lizcano, J.M. and Cuenda, A. (2010) Alternative ERK5 regulation by phosphorylation during the cell cycle. Cell. Signal. 22, 1829-1837

17 Dickinson, R.J. and Keyse, S.M. (2006) Diverse physiological functions for dual-specificity MAP kinase phosphatases. J. Cell Sci. 119, 4607-4615

18 Buschbeck, M., Eickhoff, J., Sommer, M.N. and Ullrich, A. (2002) Phosphotyrosine-specific phosphatase PTP-SL regulates the ERK5 signaling pathway. J. Biol. Chem. 277, 2950329509

19 Woo, C.H., Shishido, T., McClain, C., Lim, J.H., Li, J.D., Yang, J., Yan, C. and Abe, J. (2008) Extracellular signal-regulated kinase 5 SUMOylation antagonizes shear stressinduced antiinflammatory response and endothelial nitric oxide synthase expression in endothelial cells. Circ. Res. 102, 538-545

20 Nishimoto, S. and Nishida, E. (2006) MAPK signalling: ERK5 versus ERK1/2. EMBO Rep. 7, 782-786

21 Wang, X. and Tournier, C. (2006) Regulation of cellular functions by the ERK5 signalling pathway. Cell. Signal. 18, 753-760 
22 Hayashi, M. and Lee, J.D. (2004) Role of the BMK1/ERK5 signaling pathway: lessons from knockout mice. J. Mol. Med. 82, 800-808

23 Nithianandarajah-Jones, G.N., Wilm, B., Goldring, C.E., Muller, J. and Cross, M.J. (2012) ERK5: structure, regulation and function. Cell. Signal. 24, 2187-2196

24 Regan, C.P., Li, W., Boucher, D.M., Spatz, S., Su, M.S. and Kuida, K. (2002) Erk5 null mice display multiple extraembryonic vascular and embryonic cardiovascular defects. Proc. Natl. Acad. Sci. U.S.A. 99, 9248-9253

25 Sohn, S.J., Sarvis, B.K., Cado, D. and Winoto, A. (2002) ERK5 MAPK regulates embryonic angiogenesis and acts as a hypoxia-sensitive repressor of vascular endothelial growth factor expression. J. Biol. Chem. 277, 43344-43351

26 Yan, L., Carr, J., Ashby, P.R., Murry-Tait, V., Thompson, C. and Arthur, J.S. (2003) Knockout of ERK5 causes multiple defects in placental and embryonic development. BMC Dev. Biol. 3, 11

27 Hayashi, M., Kim, S.W., Imanaka-Yoshida, K., Yoshida, T., Abel, E.D., Eliceiri, B., Yang, Y., Ulevitch, R.J. and Lee, J.D. (2004) Targeted deletion of BMK1/ERK5 in adult mice perturbs vascular integrity and leads to endothelial failure. J. Clin. Invest. 113, 1138-1148

28 Wang, X., Merritt, A.J., Seyfried, J., Guo, C., Papadakis, E.S., Finegan, K.G., Kayahara, M., Dixon, J., Boot-Handford, R.P., Cartwright, E.J. et al. (2005) Targeted deletion of mek5 causes early embryonic death and defects in the extracellular signal-regulated kinase 5/myocyte enhancer factor 2 cell survival pathway. Mol. Cell. Biol. 25, 336-345

29 Yang, J., Boerm, M., McCarty, M., Bucana, C., Fidler, I.J., Zhuang, Y. and Su, B. (2000) Mekk3 is essential for early embryonic cardiovascular development. Nat. Genet. 24, 309313

30 Li, T., Pan, Y.W., Wang, W., Abel, G., Zou, J., Xu, L., Storm, D.R. and Xia, Z. (2013) Targeted deletion of the ERK5 MAP kinase impairs neuronal differentiation, migration, and survival during adult neurogenesis in the olfactory bulb. PLoS ONE 8, e61948

31 Lin, Q., Lu, J., Yanagisawa, H., Webb, R., Lyons, G.E., Richardson, J.A. and Olson, E.N. (1998) Requirement of the MADS-box transcription factor MEF2C for vascular development. Development 125, 4565-4574

32 Lin, Q., Schwarz, J., Bucana, C. and Olson, E.N. (1997) Control of mouse cardiac morphogenesis and myogenesis by transcription factor MEF2C. Science 276, 1404-1407

$33 \mathrm{Bi}$, W., Drake, C.J. and Schwarz, J.J. (1999) The transcription factor MEF2C-null mouse exhibits complex vascular malformations and reduced cardiac expression of angiopoietin 1 and VEGF. Dev. Biol. 211, 255-267

34 Olson, E.N. (2004) Undermining the endothelium by ablation of MAPK-MEF2 signaling. J. Clin. Invest. 113, 1110-1112

35 Roberts, O.L., Holmes, K., Muller, J., Cross, D.A. and Cross, M.J. (2010) ERK5 is required for VEGF-mediated survival and tubular morphogenesis of primary human microvascular endothelial cells. J. Cell Sci. 123, 3189-3200

36 Wang, X., Finegan, K.G., Robinson, A.C., Knowles, L., Khosravi-Far, R., Hinchliffe, K.A., Boot-Handford, R.P. and Tournier, C. (2006) Activation of extracellular signal-regulated protein kinase 5 downregulates FasL upon osmotic stress. Cell Death Differ. 13, 2099-2108

37 Finegan, K.G., Wang, X., Lee, E.J., Robinson, A.C. and Tournier, C. (2009) Regulation of neuronal survival by the extracellular signal-regulated protein kinase 5 . Cell Death Differ. 16, 674-683

38 Lennartsson, J., Burovic, F., Witek, B., Jurek, A. and Heldin, C.H. (2010) Erk 5 is necessary for sustained PDGF-induced Akt phosphorylation and inhibition of apoptosis. Cell. Signal. 22, 955-960

39 Razumovskaya, E., Sun, J. and Ronnstrand, L. (2011) Inhibition of MEK5 by BIX02188 induces apoptosis in cells expressing the oncogenic mutant FLT3-ITD. Biochem. Biophys. Res. Commun. 412, 307-312

40 Jacinto, E., Facchinetti, V., Liu, D., Soto, N., Wei, S., Jung, S.Y., Huang, Q., Qin, J. and Su, B. (2006) SIN1/MIP1 maintains rictor-mTOR complex integrity and regulates Akt phosphorylation and substrate specificity. Cell 127, 125-137 
41 Sarbassov, D.D., Ali, S.M., Sengupta, S., Sheen, J.H., Hsu, P.P., Bagley, A.F., Markhard, A.L. and Sabatini, D.M. (2006) Prolonged rapamycin treatment inhibits mTORC2 assembly and Akt/PKB. Mol. Cell 22, 159-168

42 Alessi, D.R., Andjelkovic, M., Caudwell, B., Cron, P., Morrice, N., Cohen, P. and Hemmings, B.A. (1996) Mechanism of activation of protein kinase B by insulin and IGF-1. EMBO J. 15, 6541-6551 PubMed

43 Pankov, R., Cukierman, E., Clark, K., Matsumoto, K., Hahn, C., Poulin, B. and Yamada, K.M. (2003) Specific $\beta 1$ integrin site selectively regulates Akt/protein kinase B signaling via local activation of protein phosphatase 2A. J. Biol. Chem. 278, 18671-18681

44 Razmara, M., Eger, G., Rorsman, C., Heldin, C.H. and Lennartsson, J. (2012) MKP3 negatively modulates PDGF-induced Akt and Erk5 phosphorylation as well as chemotaxis. Cell. Signal. 24, 635-640

45 Nigro, P., Abe, J. and Berk, B.C. (2011) Flow shear stress and atherosclerosis: a matter of site specificity. Antioxid. Redox Signal. 15, 1405-1414

46 Berk, B.C. (2008) Atheroprotective signaling mechanisms activated by steady laminar flow in endothelial cells. Circulation 117, 1082-1089

47 Pi, X., Garin, G., Xie, L., Zheng, Q., Wei, H., Abe, J., Yan, C. and Berk, B.C. (2005) BMK1/ERK5 is a novel regulator of angiogenesis by destabilizing hypoxia inducible factor 1a. Circ. Res. 96, 1145-1151

48 Akaike, M., Che, W., Marmarosh, N.L., Ohta, S., Osawa, M., Ding, B., Berk, B.C., Yan, C. and Abe, J. (2004) The hinge-helix 1 region of peroxisome proliferator-activated receptor $Y$ 1 (PPARY 1) mediates interaction with extracellular signal-regulated kinase 5 and PPARY 1 transcriptional activation: involvement in flow-induced PPAR $y$ activation in endothelial cells. Mol. Cell. Biol. 24, 8691-8704

49 Li, L., Tatake, R.J., Natarajan, K., Taba, Y., Garin, G., Tai, C., Leung, E., Surapisitchat, J., Yoshizumi, M., Yan, C. et al. (2008) Fluid shear stress inhibits TNF-mediated JNK activation via MEK5-BMK1 in endothelial cells. Biochem. Biophys. Res. Commun. 370, 159-163

50 Dekker, R.J., van Soest, S., Fontijn, R.D., Salamanca, S., de Groot, P.G., VanBavel, E., Pannekoek, H. and Horrevoets, A.J. (2002) Prolonged fluid shear stress induces a distinct set of endothelial cell genes, most specifically lung Krüppel-like factor (KLF2). Blood 100, 1689-1698

51 SenBanerjee, S., Lin, Z., Atkins, G.B., Greif, D.M., Rao, R.M., Kumar, A., Feinberg, M.W., Chen, Z., Simon, D.I., Luscinskas, F.W. et al. (2004) KLF2 Is a novel transcriptional regulator of endothelial proinflammatory activation. J. Exp. Med. 199, 1305-1315

52 Boon, R.A. and Horrevoets, A.J. (2009) Key transcriptional regulators of the vasoprotective effects of shear stress. Hamostaseologie 29, 39-40, 41-33 PubMed

53 Dekker, R.J., Boon, R.A., Rondaij, M.G., Kragt, A., Volger, O.L., Elderkamp, Y.W., Meijers, J.C., Voorberg, J., Pannekoek, H. and Horrevoets, A.J. (2006) KLF2 provokes a gene expression pattern that establishes functional quiescent differentiation of the endothelium. Blood 107, 4354-4363

54 Sohn, S.J., Li, D., Lee, L.K. and Winoto, A. (2005) Transcriptional regulation of tissuespecific genes by the ERK5 mitogen-activated protein kinase. Mol. Cell. Biol. 25, 85538566

55 Parmar, K.M., Larman, H.B., Dai, G., Zhang, Y., Wang, E.T., Moorthy, S.N., Kratz, J.R., Lin, Z., Jain, M.K., Gimbrone, Jr, M.A. and Garcia-Cardena, G. (2006) Integration of flowdependent endothelial phenotypes by Krüppel-like factor 2. J. Clin. Invest. 116, 49-58

56 Slater, S.C., Ramnath, R.D., Uttridge, K., Saleem, M.A., Cahill, P.A., Mathieson, P.W., Welsh, G.I. and Satchell, S.C. (2012) Chronic exposure to laminar shear stress induces Krüppel-like factor 2 in glomerular endothelial cells and modulates interactions with cocultured podocytes. Int. J. Biochem. Cell Biol. 44, 1482-1490

57 Lin, Z., Kumar, A., SenBanerjee, S., Staniszewski, K., Parmar, K., Vaughan, D.E., Gimbrone, Jr, M.A., Balasubramanian, V., Garcia-Cardena, G. and Jain, M.K. (2005) Krüppel-like factor 2 (KLF2) regulates endothelial thrombotic function. Circ. Res. 96, e4857 
58 Ohnesorge, N., Viemann, D., Schmidt, N., Czymai, T., Spiering, D., Schmolke, M., Ludwig, S., Roth, J., Goebeler, M. and Schmidt, M. (2010) Erk5 activation elicits a vasoprotective endothelial phenotype via induction of Krüppel-like factor 4 (KLF4). J. Biol. Chem. 285, 26199-26210

59 Clark, P.R., Jensen, T.J., Kluger, M.S., Morelock, M., Hanidu, A., Qi, Z., Tatake, R.J. and Pober, J.S. (2011) MEK5 is activated by shear stress, activates ERK5 and induces KLF4 to modulate TNF responses in human dermal microvascular endothelial cells. Microcirculation 18, 102-117

60 Kim, M., Kim, S., Lim, J.H., Lee, C., Choi, H.C. and Woo, C.H. (2012) Laminar flow activation of ERK5 protein in vascular endothelium leads to atheroprotective effect via NFE2-related factor 2 (Nrf2) activation. J. Biol. Chem. 287, 40722-40731

61 Bryan, H.K., Olayanju, A., Goldring, C.E. and Park, B.K. (2013) The Nrf2 cell defence pathway: Keap1-dependent and -independent mechanisms of regulation. Biochem. Pharmacol. 85, 705-717

62 Folkman, J. (2007) Angiogenesis: an organizing principle for drug discovery? Nat. Rev. Drug Discov. 6, 273-286 CrossRef

63 Holmes, K., Roberts, O.L., Thomas, A.M. and Cross, M.J. (2007) Vascular endothelial growth factor receptor-2: structure, function, intracellular signalling and therapeutic inhibition. Cell. Signal. 19, 2003-2012

64 Hayashi, M., Fearns, C., Eliceiri, B., Yang, Y. and Lee, J.D. (2005) Big mitogen-activated protein kinase 1/extracellular signal-regulated kinase 5 signaling pathway is essential for tumor-associated angiogenesis. Cancer Res. 65, 7699-7706 PubMed

65 Tatake, R.J., O’Neill, M.M., Kennedy, C.A., Wayne, A.L., Jakes, S., Wu, D., Kugler, Jr, S.Z., Kashem, M.A., Kaplita, P. and Snow, R.J. (2008) Identification of pharmacological inhibitors of the MEK5/ERK5 pathway. Biochem. Biophys. Res. Commun. 377, 120-125

66 Yang, Q., Deng, X., Lu, B., Cameron, M., Fearns, C., Patricelli, M.P., Yates, 3rd, J.R., Gray, N.S. and Lee, J.D. (2010) Pharmacological inhibition of BMK1 suppresses tumor growth through promyelocytic leukemia protein. Cancer Cell 18, 258-267 


\section{Figure Legends:}

Figure 1: The ERK5 signalling axis

ERK5 is activated by a linear signalling cascade. MEKK2/MEKK3 (MAPKKK) phosphorylate MEK5 (MAPKK), which in turn phosphorylates ERK5 (MAPK) on Thr218 and Tyr220 within the activation loop of the kinase domain. The ERK5 protein consists of 816 amino acids and contains a kinase domain, NLS, two proline-rich domains (PR1 and PR2) and a relatively large C-terminal tail of approximately 400 amino acids. Activation of ERK5 facilitates its nuclear localization and phosphorylation of a range of substrates such as MEF2C, c-Myc, Ets domain transcription factor serum-response factor accessory protein 1a (Sap-1), SGK, p90 RSK (reviewed in [21,22]). CSF-1, colony-stimulating factor 1; EGF, endothelial growth factor; LIF, leukaemia-inhibitory factor; NGF, nerve growth factor.

Figure 2: ERK5 and vascular effects

The ERK5 signalling cascade is activated in vascular endothelium in response to flow-induced shear stress and VEGF-A activation of VEGF receptor 2 (VEGFR-2). Blood flow in the normal endothelium results in shear stress due to activation of mechanosensitive channels on the apical surface of endothelial cells. Activation of MEK5/ERK5 results in increased expression of the transcription factors KLF2 and KLF4 which regulate an anti-inflammatory reaction via inhibition of NF- $K B$. Activation of KLF2 and KLF4 is also known to up-regulate expression of eNOS and thrombomodulin (TM), which results in vasoprotection. Activation of MEK5/ERK5 in response to shear stress also activates NRF2, leading to increased expression of the antioxidant genes haem oxygenase $1(\mathrm{HO}-1)$ and $\mathrm{NAD}(\mathrm{P}) \mathrm{H}$ dehydrogenase (quinone) 1 (NQO-1). VEGFR-2-mediated activation of MEK5/ERK5 results in phosphorylation of PKB/Akt in endothelial cells, which leads to increased expression of BCL-2 and increased phosphorylation of BAD, resulting in cell survival. 


\section{Figure 1}

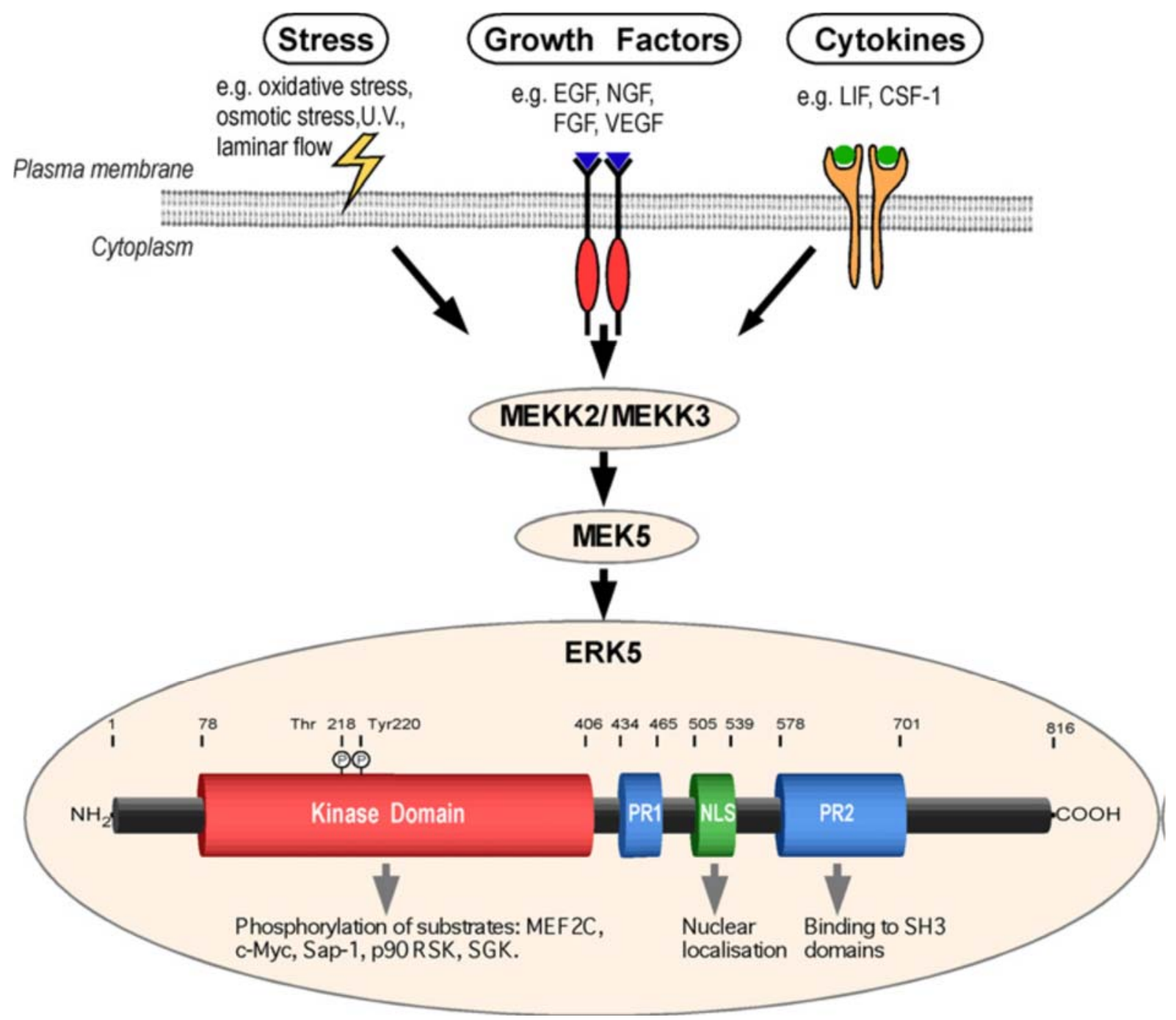




\section{Figure 2}

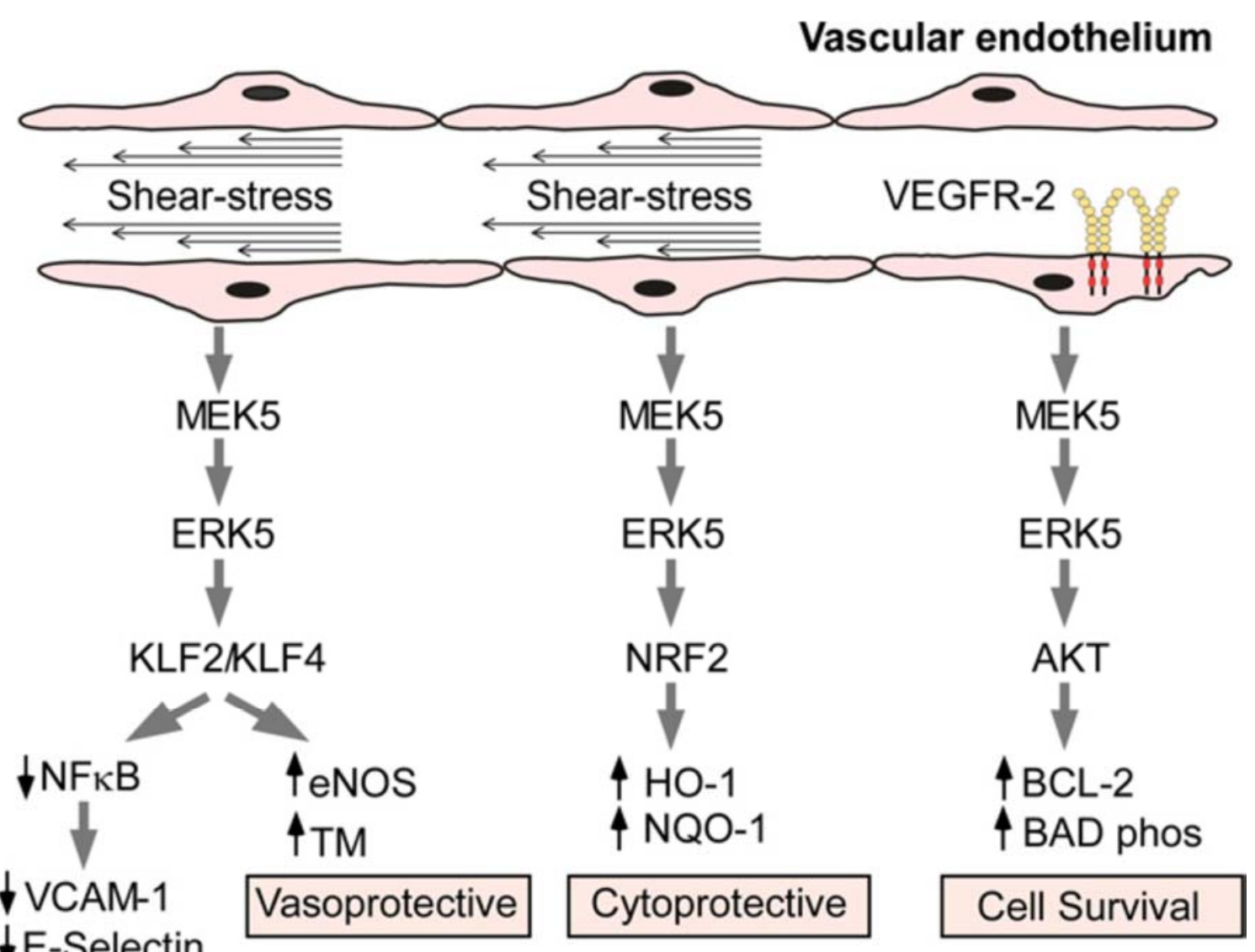

Anti-inflammatory 